\title{
Adsorption of water on epitaxial graphene
}

\author{
U. Burghausa \\ Department of Chemistry and Biochemistry, North Dakota State University, Fargo, North Dakota 58108-6050, USA \\ a)e-mail: uwe.burghaus@ndsu.edu; www.uweburghaus.us/ \\ Received: 26 May 2020; accepted: 16 July 2020; published online: 4 February 2021
}

Graphene and its functionalization are still one of the most prominent two-dimensional crystals. In recent years, the wetting properties of graphene for water (i.e., its hydrophobic, hydrophilic, and also icophobic features) were controversially discussed as well as water intercalation and confined water, that have unusual characteristics. The dispute about wetting properties was originally based on contact angle (/engineering) measurements conducted at ambient pressure. In the meanwhile, detailed ultra-high vacuum (UHV) surface science works and theoretical studies are available. This brief review describes the current knowledge available in the literature about the water/graphene system as well as our own work using experimental UHV surface science techniques. The review starts with a definition of hydrophobicity and briefly touches on a possible correlation with icephobicity as well as discusses briefly confined water. Next, theoretical studies are reviewed, and finally, experimental works are described on which the review focusses. Finally, a brief outlook section discusses water adsorption on functionalized graphene.

\section{INTRODUCTION}

In regard to basic science and applications, two-dimensional (2D) crystals are especially important for the materials research community. In particular, gas-surface interactions are pertinent for sensors and catalysis, as well as materials science and surface science in general. Basic science of $2 \mathrm{D}$ crystals is devoted to a better understanding of fundamental differences of 2D crystals and bulk materials, support effects for epitaxially grown materials, and control of surface reactivity among other properties. The gained knowledge will support diverse applications in environmental and energy technologies as well as nanoelectronics. Still, graphene and its functionalization are a most prominent $2 \mathrm{D}$ crystal. In recent years, the wetting properties of graphene for water were controversially discussed as well as water intercalation and confined water with unusual properties. The dispute about wetting properties was originally based on contact angle (/engineering) measurements conducted at ambient pressure. In the meanwhile, detailed surface science works including theoretical studies are available. For biological surfaces such as plant leaves, a combination of micro- and nanoscale morphologies seem to enhance hydrophobicity (lotus effect) which motivates many studies on nanostructured surfaces [1]. The many also commercial applications of hydrophobic surfaces become even more pertinent since it seems that hydrophobic surfaces are also icephobic [2].

This brief review describes the current knowledge available in the literature about water/graphene as well as our own work $[3,4,5,6,7,8,9,10,11,12]$ using ultra-high vacuum (UHV) surface science techniques. The review focuses on a discussion of the wetting properties and experimental projects, but some studies about the morphology, kinetics, and electronic structure are also included as far as these concern water adsorption. This review is specialized on water/graphene. Gas-surface interactions on $2 \mathrm{D}$ crystals, in general, were discussed in a recent longer review [13], as well as for water adsorption on graphene/silicatene see [6] and for water/nanotubes see [14]. Most reviews about 2D materials still emphase the synthesis of these materials. This article's focus is on properties and the characterization of materials by emphasizing concepts over the very details. For other reviews about water surface chemistry, see Refs. [6,15,16,17].

\section{WHAT IS A HYDROPHOBIC OR HYDROPHILIC SURFACE?}

\section{Contact angles defining wettability}

Since this arcticle's focus is on wetting properties of graphene, a logical starting point is providing a quick definition. Figure 1 
(a)

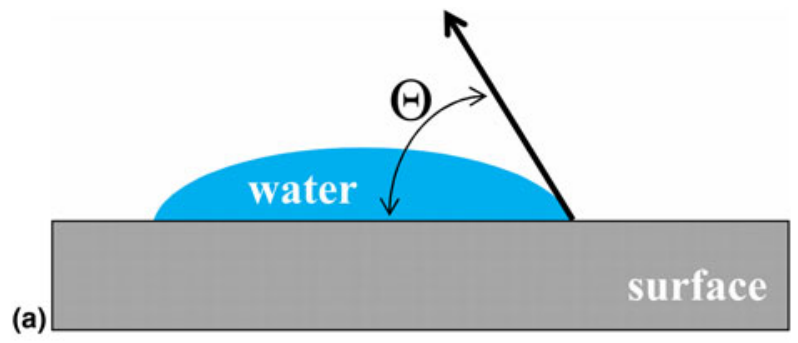

Hydrophobic

$\Theta \gg 90^{\circ}$ (usually $80-85^{\circ}$ )

Superhydrophobic

Hydrophilic

(b)Superhydrophilic

$\Theta=180^{\circ}$

$\Theta<<90^{\circ}$ (usually $40-60^{\circ}$ )

$\Theta=0^{\circ}$

Figure 1: (a) Schematics of a water droplet on a surface and (b) common definition of the contact angle.

shows a water droplet on a surface (here graphene) and how the contact angle is usually defined [18,19]. Depending on the numerical value of that angle, a surface is called hydrophobic (nonwetting) or hydrophilic (wetting). Later described is how to determine this property experimentally and how to model it. In surface science, the terms wetting versus nonwetting also are used, for e.g., metals deposited on surfaces (so-called model catalyst) depending on what (cluster) morphology is formed [20]. A nonwetting surface should result in the droplet (/cluster) formation, whereas wetting surfaces promote the formation of closed thin film-like structures. Whether or not that happens can be described using the interface energy (energy required to separate an interface), a thermodynamics approach. Three-dimensional (3D) cluster growth (droplets) is also denoted as Volmer-Weber growth related to nonwetting systems and, e.g., metal deposits. Thin film formation would be a layer-by-layer growth or a Frank-van der Merwe morphology, using surface science methodology. There is also a morphology in-between these extreme cases such as layers plus islands growth modes, also called Stranski-Krastanov growth of adsorbates on a surface. For details on this, see, e.g., Ref. [20]. This is mentioned here since materials science is interdisciplinary where colleagues often use different terms that basically describe the same phenomena.

\section{Binding energies defining wettability}

Despite contact angles, what other criteria that are experimentally accessible could be used to define wetting properties? For example, inert surfaces with small binding energies for adsorbates should be hydrophobic. Or, if water molecules in a water droplet (/cluster) bind stronger together than the droplet binds to the surface, that surface should be hydrophobic. On the other extreme, large binding energies (high reactivity) should correspond to hydrophilic surfaces. The problem, however, is what actually is large or small? In some molecular dynamics (MD) simulations [21], a surface is hydrophilic when water binds stronger than $44 \mathrm{~kJ} / \mathrm{mol}$ (heat of liquefaction) [21]. But other threshold energies have been used as well [22]. Typically, inert surfaces such as silica, silver, or gold are indeed hydrophobic. Now at the end of this short introduction, this article briefly touches on two related topics of current interest: icephobicity and confined water.

\section{Icephobicity}

Ice formation is unwanted in many applications (on wings of aircrafts, ski glasses, winter cloth, buildings, power lines, etc.) [23]. Estimates call solving that problem a 20 billion/year market [24]. Likely correlated to hydrophobicity is icephobicity, the property of a surface to resist ice formation. A review on this relatively new subject would be in ref. [24]. Ice is typically thought being formed from a nucleus consisting of a small crystallite. Therefore, one would expect that a surface that resists water adsorption also resists ice formation. Many publications deal in particular with that hypothesis [25,26,27]. For example, in some studies, with the increasing contact angle of liquid water (surface becoming more hydrophobic, Table 1), ice adhesion indeed decreases [24]. However, also other correlations were seen such as effects of surface morphologies including surface roughness, or contact angle hysteresis, or surface coatings (including liquid surface coatings, called slippery liquid infused porous surfaces [23]), etc. Arguments against a simple correlation of hydrophobicity and icephobicity consider the different mechanical properties of liquid water and ice water on surfaces. According to the data presented in Ref. [25], for good icophobicity, good surface smoothness seemed more important than hydrophobicity. Other groups too reject a direct correlation of water wetting and ice formation [27]. A brief outline of the complex thermodynamics can be found in Ref. [24] which also discusses how to actually quantify icephobicity. A simple experimental approach to do so is using the freezing delay time on surfaces [25].

Usually all these "phobicity" terms historically refer to water but have also been used more generally. For example, Wang and Wang [33] discuss superoleophobic membranes, i.e., surfaces that reject oil with the potential to separate oil and water. Or, the term lipophobicity is in use for fat rejection, as water itself does.

\section{Confined water}

For example, SciFinder in May 2020 provides 2000 hits using the search term "confined water" with 50 publications in spring 2020 alone. The idea is to study water confined, e.g., between a graphene layer (or other 2D materials) and a substrate or water in graphene oxide (GO) nanopores or water inside of micelles, as well as water inside of nanotubes. The latter was already 
TABLE 1: Binding energies of water on 2D crystals.

\begin{tabular}{llcl}
\hline \hline Surface & Method & Binding energy $(\mathrm{kJ} / \mathrm{mol})$ & Ref. \\
\hline Free-standing graphene & vdW-DF2 & 11.2 & {$[29]$} \\
& DFT & $1.7-4.5$ & {$[30]$} \\
& MCS & $<9.6$ & {$[31]$} \\
& DFT & $1.7-4.5$ & {$[30]$} \\
& DFT/CC & 12.8 & {$[32]$} \\
HOPG & CCSD(T) & 13.0 & {$[56]$} \\
\hline \hline
\end{tabular}

DFT, density functional theory; vdW-DF2, DFT using functionals specialized to describe van der Waals interactions; MCS, Monte Carlo simulations; CC, coupled clusters DFT; $1 \mathrm{eV}=96.485 \mathrm{~kJ} / \mathrm{mol}$.

studied many years ago [34,35]. Of interest, are changes in the solvent properties of water, the dielectric constant, diffusion coefficients, or freezing/melting behavior, ... Generally, the question is: what is the difference of bulk water (e.g., in the center of a droplet) and water at or in-between an interface? Recent reviews on this topic could be found here, see Refs. [36,37].

One of the most amazing results is that $2 \mathrm{D}$ water films apparently show ice-like structures at room temperature (!) and ambient conditions, whereas thicker water films are fluidlike structures [38]. Ice-like being defined as an ordered water structure observed, e.g., by microscopic techniques formed due to a 2D hydrogen-bonded network and hindered diffusion. Techniques such as scanning polarization force microscopy (SPFM), atomic force microscopy (AFM), ellipsometry, and infrared (IR) spectroscopy are used. In some AFM studies at ambient pressure and room temperature, 2D water layer on a substrate were covered by graphene which is flexible enough [28] to match the morphology of the graphene-covered water layer. In so doing, high image resolution can be reached without disturbing the water structure which revealed the crystalline state of water [38].

\section{THEORETICAL STUDIES ABOUT WATER ADSORPTION ON GRAPHENE \\ Difficulties describing wetting properties theoretically}

This short review focusses on experimental works, therefore, only brief remarks about theoretical studies are given in the following. Water droplets consist of a huge number of water molecules (thousands for nano-sized droplets) [39]. Most first principle techniques (Hartree-Fock, DFT, MP2, etc.) cannot easily describe systems of that size and have difficulties to describe van der Waals interactions [40]. Therefore, many theoretical studies attempting to model water wetting properties use MD simulations [39] rather than DFT. The choice of the force fields in MD is often rather artificial which can become problematic. Depending on the parameterization used, hydrophobic or hydrophilic properties may emerge [31,41] using basically the same MD models. For a number of MD studies, see, e.g., Refs. [19,39,42,43,44,45]. For example, the MD simulations in Ref. [39] (accompanied with experiments) show that for epitaxial graphene, the wetting properties of the substrate are only partially transmitted through graphene. Contact angles for water were calculated for various substrates, e.g., $96^{\circ}$ for a hydrophobic substrate (functionalized silica) [39]. The MD simulation in Ref. [19] predicts an increase in the contact angle from $84^{\circ}$ to $93^{\circ}$ when covering clean copper with up to six graphene layers which at that point approaches the bulk value of graphite. Therefore, graphene flakes often studied in solution phase resemble properties of HOPG rather than graphene.

\section{Electronic structure, adsorption sites and configurations}

Table 1 lists binding energies determined by various DFT versions as well as by Monte Carlo simulations (MCS). The energy range in DFT usually refers to different adsorption sites or adsorption configurations. DFT usually considers a single water molecule or small water clusters on free-standing graphene. Therefore, it is difficult to compare the theoretical results directly with most experimental studies. Kinetic techniques such as thermal desorption spectroscopy (TDS) can, however, in principle provide estimates of binding energies in the low coverage limit. The table also includes data for systems that can be used as a reference for studies on graphene such as HOPG (highly ordered pyrolytic graphite).

DFT studies in the low concentration limit usually try to determine the energetically most favorable adsorption site and configuration. Widely different conclusions can be found in different DFT studies, however. For example, in Ref. [46], on-top adsorption sites with water in a down configuration (both $\mathrm{H}$ atoms pointing towards graphene) is energetically preferred [46]. In Ref. [30], hollow adsorption sites (above the center of the honeycomb of graphene) were the most stable situation.

\section{Effect of the substrate for epitaxial graphene}

In experimental surface science studies, usually epitaxial graphene is characterized rather than free-standing graphene. (On the other hand, thick graphene flakes often studied in solution are closer to bulk materials, HOPG, than graphene.) Therefore, one concern is how the choice of the substrate affects the surface properties of epitaxial graphene. Indeed, some DFT and MD studies $[47,48,49,50]$ discuss the substrate effects for epitaxial graphene. For example, how does the polarizability of the substrate affect graphene's surface properties? 
Metallic or semiconducting substrates should potentially show very different polarizabilities. Thus, the selection of the substrate may allow for tuning surface properties. These effects are still debated in computational studies. For example, the MD calculations in Ref. [39] predict only week substrate effects in contrast to very similar MD simulations in Ref. [19].

\section{WETTING PROPERTIES OF GRAPHENE FOR WATER: EXPERIMENTAL STUDIES}

\section{How can wetting properties be determined experimentally?}

Wetting properties for graphene were initially determined by measuring the contact angle (see Fig. 1) [51] using, e.g., an optical microscope/goniometer. That technique works only at ambient conditions. For reducing contaminations, in some studies, glove boxes were used, or experiments were conducted in the inert atmosphere. Alternatively, the contact angle of an air bubble on the surface submerged in water also can be measured which may lead to cleaner measuring conditions. Depending on the determined contact angle, the surface is considered hydrophobic or hydrophilic (Fig. 1).

Applying surface science methodology, the concern is whether or not $3 \mathrm{D}$ water clusters form initially or rather complete 2D layers of water. (Droplets or clusters as surface chemistry would rather write are typical for hydrophobic surfaces, whereas film formation is typical for hydrophilic surfaces.) Therefore, techniques such as scanning tunneling microscopy (STM) or AFM could theoretically be used to determine directly wetting properties [36,52]. Some variations of these methods do work in ambient air where water droplets could be studied. (Indeed, the AFM technique was used for that purpose, see Ref. [52]). However, in that case, the concern about air-borne contaminations [53] would be the same as with engineering-type contact angle measurements. Surface science microscopy techniques are UHV compatible, but liquid water droplets (/thin films) at room temperature obviously would evaporate instantly. UHV conditions would guaranty clean measuring conditions. Spectroscopic techniques (that work in ambient or UHV), such as sum-frequency generation (SFG) spectroscopy [54,55] also have been used, but these methods provide rather indirect information about wetting properties. (In SFG studies, the position of the $\mathrm{OH}$ stretch vibration is used to identify hydrophobicity [55].) A simple, commonly available and less expensive UHV compatible method that allows to determine wetting properties fairly directly at clean conditions is TDS $[3,4]$, also called temperature programmed desorption (TPD). In a TDS experiment, water would be adsorbed on the surface at low temperature in UHV where desorption (evaporation) rates are practically zero. Then, the temperature is raised (typically linearly with time) and the desorbing water detected simultaneously (usually with a mass spectrometer). A TDS curve shows the desorption rate versus surface temperature. TDS is a kinetics technique usually applied to determine experimentally binding energies. However, in some cases, TDS also does provide information about adsorption morphologies. The clue here is to realize that zeroth-order kinetics is closely related to a hydrophobic surface. That type of kinetics can be distinguished from other desorption orders rather easily.

\section{Determining wetting properties with a kinetics technique}

The proposed hypothesis is that a hydrophobic surface shows zeroth-order kinetics. One can verify that empirically by conducting a literature search, see Table 2. But, why is that the case? Remembering basic kinetics taught in a Pchem class: that kinetics is independent of the concentration which makes the desorption rate in a TDS experiment coverage independent (as long as some water droplets are left). Therefore, it is easy to verify zeroth-order kinetics experimentally. A rate independent of (starting) coverage results in a common low temperature leading edge in a set of TDS data collected for different starting coverages. In other words, all the TDS curves overlap at low temperatures independent of the starting concentration.

However, why would a hydrophobic surface shows concentration independent desorption? Practically, kinetics becomes coverage independent when a large reservoir of some sort is involved, e.g., water desorption from an ocean. The loss of water from the ocean is so small compared with the oceans' size that the water amount (/concentration) practically does not change. That makes the desorption rate become concentration-independent. Are water droplets on a hydrophobic surface an ocean? Not really, but one can simulate an ocean effect by establishing a two-phase regime. One phase is a reservoir and the other phase is driving the desorption. In that case, desorption from one phase (the active one that desorbs the water) becomes independent of concentration, as long as the reservoir (the second phase) is not depleted. Water evaporates (/desorbs) from the outer surface of a droplet in ambient (or in vacuum). The droplet (or 3D cluster) shrinks due to desorption, but the surface is reformed simultaneously during the desorption from the interior of the droplet. So, we do have a two-phase system, the surface and the interior of the droplet. That desorption becomes coverage independent as long as the interior is large enough to resupply the surface. And the diffusion to the surface is faster than the desorption which usually is the case. Figure 2 illustrates that model. 
TABLE 2: Experimental results of water adsorption on various clean and functionalized surfaces which may act as a reference for graphene surfaces.

\begin{tabular}{|c|c|c|c|c|c|}
\hline System & Method & Hydrophobic & $\begin{array}{l}\text { Zeroth- } \\
\text { order } \\
\text { kinetics? }\end{array}$ & $\begin{array}{l}\text { Number } \\
\text { of TDS } \\
\text { peaks }\end{array}$ & Ref. \\
\hline $\mathrm{Au}(111)$ & UHV, TDS & Yes & Yes & 1 & [59] \\
\hline $\mathrm{O}_{2}-\mathrm{Au}(111)$ & UHV, TDS & Yes & Yes & 1 & [69] \\
\hline $\mathrm{D}_{2}-\mathrm{Ni}(111)$ & UHV, TDS & Yes & Yes & 1 & [77] \\
\hline $\mathrm{D}_{2}-\operatorname{Pt}(533)$ & UHV, TDS & Yes & Yes & 1 & [78] \\
\hline Octane-Pt(111) & UHV, TDS & Yes & Yes & 1 & [65] \\
\hline Water-Pt(111) & UHV, TDS & Yes & Yes & 1 & [79] \\
\hline Antimony(111) & UHV, TDS & Yes & Yes & 1 & {$[5]$} \\
\hline $\mathrm{Cu}(111)$ & UHV, TDS & Yes & Yes & 1 & [66] \\
\hline $\mathrm{Ag}(011)$ & UHV, HREELS & & Yes & - & [80] \\
\hline Mica & & No & & & [36] \\
\hline $\mathrm{MoS}_{2}$ & & No & & & [36] \\
\hline HOPG & UHV, TDS & No & No & 4 & [81] \\
\hline HOPG & UHV, TDS & No & Yes & 1 & {$[57]$} \\
\hline HOPG & $\begin{array}{l}\text { UHV, TOF- SIMS, } \\
\text { TDS }\end{array}$ & No & No & 3 & [82] \\
\hline HOPG & $\begin{array}{l}\text { Ambient, contact } \\
\text { angle }\end{array}$ & Yes & - & & [57] \\
\hline HOPG & Ambient, AFM & Yes & & & [52] \\
\hline
\end{tabular}

UHV, ultra-high vacuum; TDS, thermal desorption spectroscopy; HREELS, high-resolution electron energy-loss spectroscopy; TOF, time-of-flight spectroscopy; SIMS, secondary ions mass spectroscopy; $\mathrm{AFM}$, atomic force microscopy; e.g., $\mathrm{O}_{2}-\mathrm{Au}(111)$ gold surface preadsorbed with $\mathrm{O}_{2}$, i.e., functionalized with $\mathrm{O}_{2}$.

The idea is not new, of course, it has been discussed, e.g., in a paper from Kasemo et al. in the 1990s [57,58]. In the same study, it was already recognized that carboneous surfaces are prone to air-borne impurities which alters their wetting properties. If we have $3 \mathrm{D}$ water cluster (droplets), a two-phase regime is formed and the kinetics becomes zeroth order. The clue is that zeroth-order kinetics is observed down to the submonolayer concentration regime (corresponds usually to below about two Langmuir water exposure). Obviously, a thick water layer on a surface would also show zeroth-order kinetics, for the same reason than for the droplets, but that zeroth-order kinetics breaks down when only a monolayer thick water film is left (no two-phase regime anymore). At that point, the zeroth-order kinetics from the thick film converts to first-order kinetics (at monolayer coverage) which results in TDS data that look quite different from the droplets case. (Below actual experimental data are discussed.) Therefore, one has to find zerothorder kinetics down to the zero coverage limit to conclude water cluster formation and a hydrophobic surface $[3,4,5,59$, $60]$. In case of a hydrophilic surface, a combination of firstorder kinetics (up to a monolayer concentration) and zerothorder condensation kinetics (at large concentration) would be expected $[15,16,17,60]$. Figure 3 summarizes the simple "rule" to identify a hydrophobic surface.

Many systems characterized in surface science by various different groups exhibit that behavior (see Table 2). In some cases, deviations from perfect zeroth-order kinetics are evident

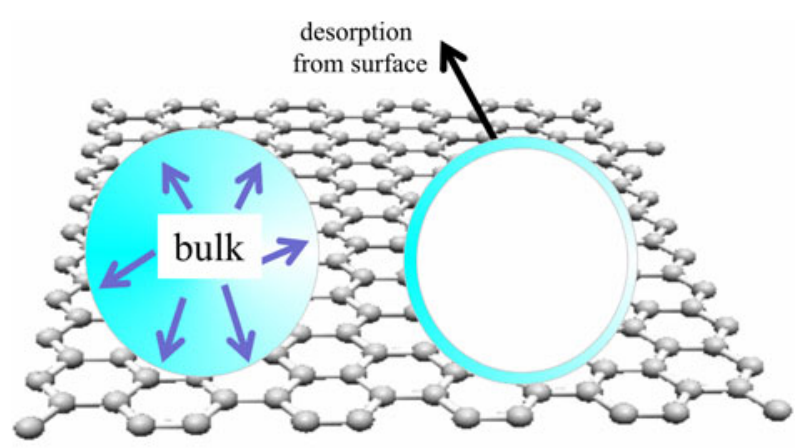

(a)

two phase regime

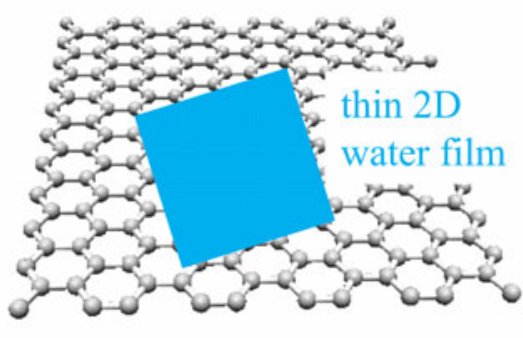

(b)

one phase regime

Figure 2: (a) Water cluster/droplets on a hydrophobic surface form a twophase regime and (b) which is absent for ultra-thin water films. Water desorbs from the surface of the droplets. Diffusion from the bulk to the surface keeps the process active and practically concentration-independent.

for systems that are considered hydrophobic [23,24]. Besides experimental reasons (i.e., limited pumping speed, readsorption, sample cleanliness, etc) coverage dependence of the kinetics due to, e.g., the effect of lateral interactions may be an explanation.

\section{Transparent versus opaque graphene and support effects}

As already noted, in experimental UHV surface science studies, epitaxial graphene is often studied, i.e., graphene is actually formed on a surface by decomposing hydrocarbons. Likewise, in engineering style experiments, transfer techniques are used to deposit graphene on various substrates. In some of these early studies, the term "transparent" graphene [19,39,61] was coined: when the wetting properties of the support match those of the supported graphene, the graphene was called transparent for water adsorption, basically implying that the graphene has no effect on its own (Fig. 4). Graphene is opaque when the hydrophobicity of the supported graphene differs from the one of the support. How can graphene become transparent? One idea is that the polarizability of the substrate is transmitted through the monoatomic graphene layer. In other words, van der Waals interactions are passed through the carbon layer $[18,39]$. Following that idea, a $100 \%$ permeability for van der Waals interactions would be needed for a fully transparent graphene film. According to one calculation 


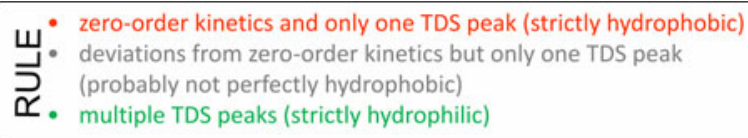

Figure 3: Simple rule to identify a hydrophobic surface with a widely available experimental tool.

[18], only $30 \%$ of the van der Waals interactions of the support are transmitted through the graphene layer, but the concept seems valid. On the other hand, it seems clear that graphene is impermeable for atoms and molecules under ambient conditions but is permeable for protons and allows electrons to tunnel through [62]. The situation changes for functionalized graphene. Thus, covalent interactions are likely blocked by the one-atom thick layer but not entirely van der Waals interactions. Not everyone agrees with the usefulness of that methodology which certainly is a simplification [51]. A related question is to what extent does the support affect the properties of graphene? Do metallic supports or semiconducting supports of graphene lead to different wetting behaviors for water? A DFT study [63] does show that graphene becomes more reactive when grown on reactive metal substrates. Defects or an intercalation may, however, also affect the conclusions. Deep mechanistic explanations of substrate effects are still mostly missing in the literature. However, clearly the electronic properties depend on the materials combination. There are a few DFT papers about CO adsorption on graphene/nickel that take the effect of the substrate into account [64] - most other DFT studies do not. For that system, apparently graphene and nickel orbitals form hybrid orbitals which alter graphene's reactivity [64]. That mechanism certainly would be specific to a given substrate and may generally explain substrate effects. Substrate effects have not been studied for a general case by DFT. Addressing the hydrophobicity directly would probably require to DFT model a water droplet with 50,000+ atoms.

\section{CONTROVERSIAL EXPERIMENTAL RESULTS OF CONTACT ANGLE MEASUREMENTS}

After all these explanations of concepts, now what is clean graphene? Is it hydrophobic or hydrophilic? It turned out that the question is trickier to answer than it seems (see Table 3). Using very similar experimental techniques often supported by theory, initially the conclusions in the literature varied wildly from total "wetting transparency of graphene" [19] to "not entirely transparent to wetting" [39] and to "negligible effect of the support" [44]. Hydrophobic and hydrophilic graphene were proposed. Most of the early studies used contact angle measurements in ambient, later on, a few groups used glove boxes and inert atmosphere for contact angle measurements and noticed then that contact angles change with measuring

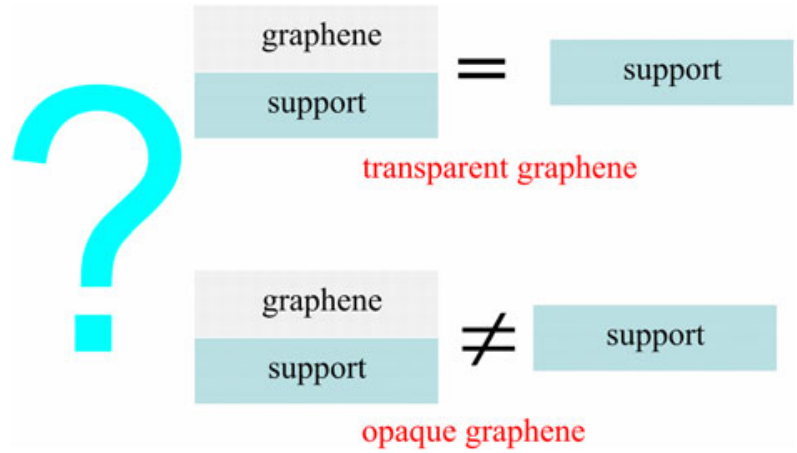

Figure 4: Does the support affect graphene's properties?

time [51,53]. Actually, at least for $\operatorname{HOPG}[37,38,47]$, it is known since the 1990s that carboneous surfaces can easily be contaminated in ambient [67]. A number of species can be generated on surfaces at ambient in various ways that may result in, e.g., hydrophilic adsorption sites [21,68]. In some cases, the formation of the hydrophilic sites may be defect mediated. Considering the electronegativity of species commonly adsorbed on surfaces, hydrogen bonding is present between water and, e.g., $\mathrm{OH}$-terminated/contaminated surfaces. (For example, surface- $\mathrm{O}^{-\epsilon} \mathrm{H} \ldots \mathrm{H}_{2}^{+\varepsilon}-\mathrm{O}$ or surface- $\mathrm{O}^{-\epsilon} \ldots$ $\mathrm{H}_{2}{ }^{+\varepsilon}$-O.) Similarly to H-terminated surfaces (e.g., surface- $\mathrm{H}^{+\epsilon} \ldots \mathrm{H}_{2} \mathrm{O}^{-\epsilon}$, etc.). The situation can become quite complex due to coverage dependent changes in the morphology of coadsorption systems, see, e.g., Ref. [69], that also can affect wetting properties in a nontrivial fashion. Similarly, a contamination such as oil (from our skin or pump exhaust in a lab) can make surfaces hydrophobic. Generally, it is impossible keeping a surface clean at ambient pressure. For example, collecting X-ray photoelectron spectroscopy (XPS) scans of a surface transferred through air usually results in the detection of a significant carbon peak due to decomposition of impurities [70]. (Some surfaces-gold, silver, mica-stay cleaner than others, but ...) That carbon is amorphous carbon which certainly changes the intrinsic properties of crystalline graphene. Another concern is studies (usually STM works) where water seems to intercalate graphene [50]. We have not seen indications for that in our own UHV TDS experiments, however [3]. One would assume that TDS data would hardly be reproducible if water intercalates and probably destroys the graphene layer [50]. Unfortunately, we did not collect AES/XPS spectra before/after water TDS experiments.

\section{Kinetics experiments for water on graphene}

In our own works, we did apply the TDS method as described above. We worked on a (monoatomic) graphene layer grown at UHV conditions on $\mathrm{Ru}(0001)$ as well as used commercial samples (graphene/ $\mathrm{SiO}_{2}$ and graphene/Cu) which were made at 
TABLE 3: Water adsorption on graphene.

\begin{tabular}{|c|c|c|c|c|c|c|c|}
\hline Substrate & Method & Transparent & Ref. & Substrate & Method & Transparent & Ref. \\
\hline \multicolumn{4}{|c|}{ Hydrophilic graphene } & \multicolumn{4}{|l|}{ Hydrophobic graphene } \\
\hline Silica & TDS & No & [4] & $\mathrm{Ru}$ & TDS & No & [3] \\
\hline $\mathrm{Cu}$ & a & Partial & {$[51]$} & $\mathrm{Cu}$ & TDS & No & [4] \\
\hline $\mathrm{Ni}$ & $a$ & Partial & [51] & Sapphire & SFG & No & [54] \\
\hline HOPG & $a$ & Partial & {$[51]$} & Pt & FTIR & - & [84] \\
\hline Silica & $a$ & No & [39] & Silica (functionalized) & a & No & [39] \\
\hline Silica & a & Yes & {$[18]$} & $\mathrm{Au}$ & $a$ & Yes & [19] \\
\hline \multirow[t]{4}{*}{$\mathrm{Cu}$} & XPS, FTIR, a & & [18] & $\mathrm{Cu}$ & $a$ & Yes & [19] \\
\hline & & & & Silica & $a$ & & [85] \\
\hline & & & & $\mathrm{CF}_{4}$-silica & a & & [85] \\
\hline & & & & Sapphire & SFG & & [54] \\
\hline
\end{tabular}

TDS, thermal desorption spectroscopy; SFG, sum-frequency generation; FTIR, Fourier transform infrared spectroscopy; $\alpha$, contact angle measurements.

ambient conditions and transferred through air to our lab $[3,4]$. We did not have the techniques available to characterize the morphology of these samples in great detail which is in particular problematic for the commercial samples transferred through air. Thus, our best data (the cleanest data) are from the graphene/Ru(0001) system that never left the vacuum and did also show a clear low-energy electron diffraction (LEED) pattern indicative of a good surface morphology [3, 4]. Regarding the substrates we used, from older studies it is known that $\mathrm{Ru}$ and $\mathrm{Cu}$ are hydrophilic but clean silica is hydrophobic. For graphene/Ru, we saw only one TDS peak (Fig. 5) at low temperature consistent with molecular water adsorption; the low-temperature edges of the TDS curves lined up down to submonolayer concentration ( $<2$ Langmuir) which is consistent with a hydrophobic surface as outlined in the beginning, but the high-temperature edges did not drop fast to zero as would be expected for perfect zeroth-order kinetics. We could rule out that the pumping speed of our setup did unlikely caused this effect. Therefore, we did conclude that graphene/Ru is fairly hydrophobic, but probably not perfectly hydrophobic. Here, the substrate and graphene on that substrate show different wetting properties, i.e., graphene/ $\mathrm{Ru}$ is not transparent for water adsorption.

For the commercial samples, we concluded hydrophilic graphene/ $\mathrm{SiO}_{2}$ and hydrophobic graphene/Cu. Therefore, we have to assume that graphene is not transparent to water wetting when grown on copper and silica supports. However, the support did affect graphene's surface properties. In some cases, we also characterized the support used to grow graphene (see Fig. 6). In the case of the silica support, one can nicely see the difference of a hydrophobic and hydrophilic surface, as seen with TDS. Graphene on silica shows a first-order desorption peak at low exposures (submonolayer range) that shifts to lower desorption temperatures with increasing exposure. Once the surface is saturated with water and thicker water layer start to form, the TDS peak shifts the direction and now moves to larger desorption temperatures with increasing exposure.
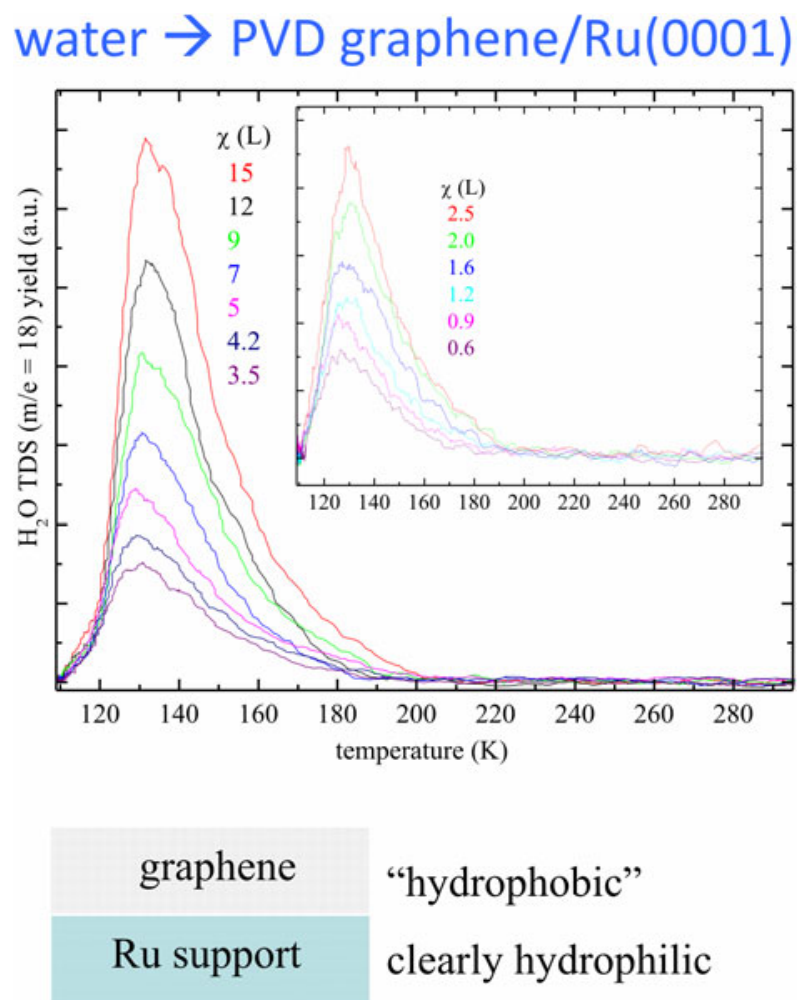

Figure 5: TDS results of water for physical vapor deposited graphene on ruthenium. The curves correspond to different initial water exposures given in Langmuir ( $1 \mathrm{~s}$ water exposure at $1 \times 10^{-6}$ torr). That the low-temperature edges of all curves line up verifies that the desorption rate is coverage independent [3].

That is typical for zeroth-order kinetics of thick condensed films. In contrast, for the bare silica, only one TDS peak is seen over the entire concentration range that always shifts to greater temperature with increasing exposure. The lowtemperature edges of all TDS traces line up, i.e., the desorption rate is independent of the (starting) coverage. Thus, silica alone is indeed hydrophobic. Silica is expected to be hydrophobic. Silicon has low-energy empty $d$ orbitals and oxygen has nonbonding electron pairs in $p$ or $s p^{n}$ hybrid orbitals. This can 


\section{water $\rightarrow$ CVD graphene/silica wafer}

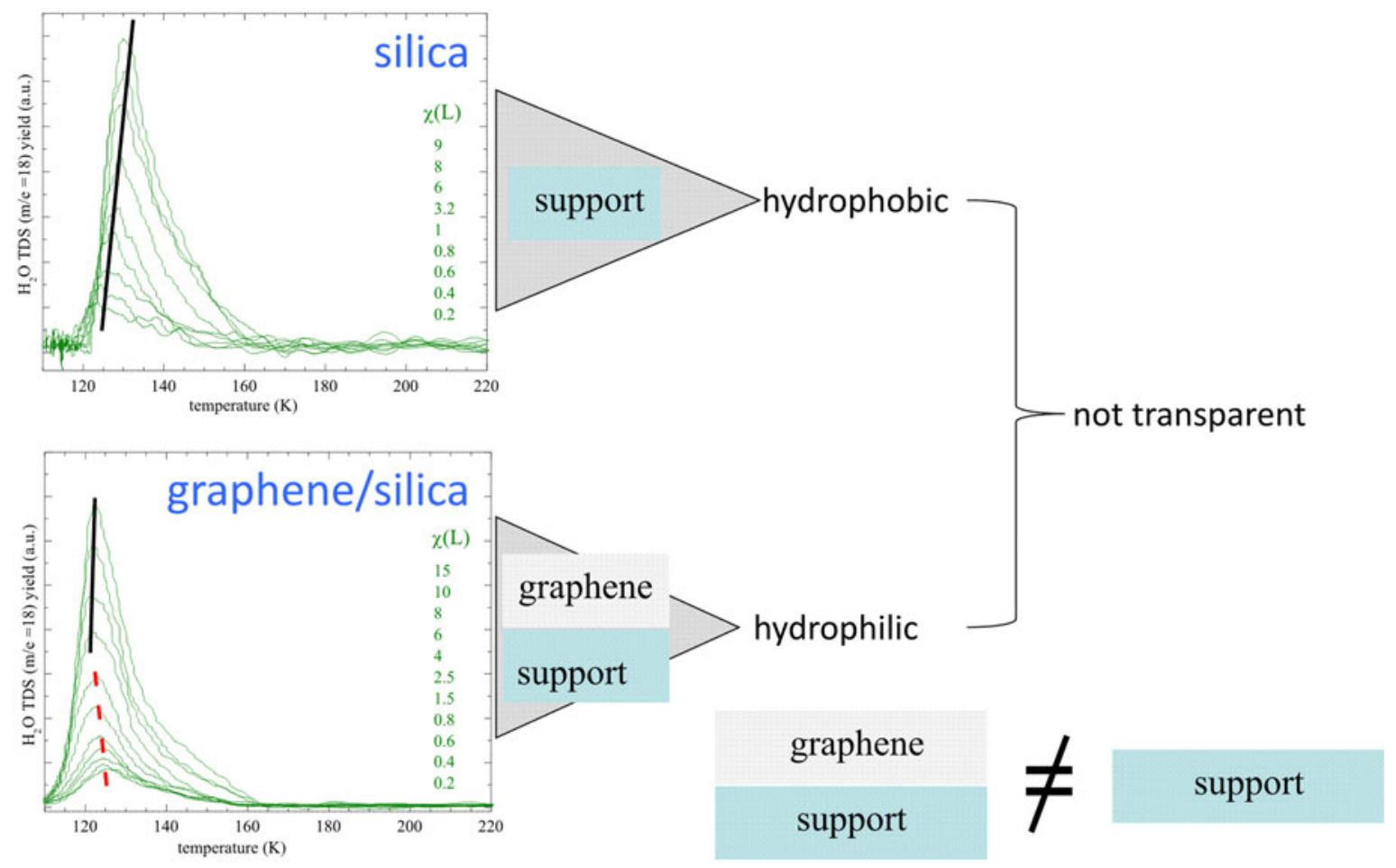

Figure 6: TDS results for water on commercial graphene-on-silica compared with the bare substrate [4].

result in partial $\pi$ bonding character in which filled $p$ or $s p^{n}$ electrons of oxygen will be shared with empty $d$ orbitals of silicon. Due to this partial $\pi$ bond character, the $\mathrm{Si}-\mathrm{O}$ bond length is shorter and significantly stronger than expected. Therefore, Si-O-Si bonds are inert and hydrophobic, see, e.g., Refs. [21,71]. Note that the cleaning procedure of this commercial graphene on the silica sample was limited and the morphology was not perfectly known which also may have affected the results. The switch from first- to zeroth-order desorption for graphene/silica happens at about 2.5 Langmuir water exposure which is typical for the formation of a water double layer and an adsorption probability of water close to one. If water intercalates easily, one would expect larger water exposures for saturating the surface/subsurface.

\section{Outlook: Water adsorption on functionalized graphene such as graphene oxide}

One of the most popular and perhaps even at UHV experimentally relatively simple to realize functionalization of graphene is GO [8]. Due to the formation of hydrogen bonds between adsorbed water and GO, one would expect a hydrophilic surface which indeed seems to be the case, see Ref. [72]. Unfortunately, a detailed UHV surface science study about water/GO wetting properties is not yet available. A number of applied works in solution phase and ambient have been conducted devoted to quantify the uptake of water by GO $[72,73]$. The desiccant (i.e., water drying) properties are praised and characterized for various applications, including food preservation [72]. Most intriguing perhaps is GO's superpermeability for water and its stability in water which again still lacks much of basic surface science research [74,75]. GO also is studied as an additive to cement where small concentrations of GO accelerate the hydration rate. The structure and properties of concrete apparently dependent on hydration reactions of concretes' components [76].

Related to GO and water adsorption is the interaction of graphene with oxygen and hydrogen which was recently reviewed in Ref. [13]. UHV surface science studies about water- $\mathrm{O}_{2}$ or water-H coadsorption have not been published. Much literature is visible about graphene functionalization besides GO, notably $\mathrm{N}$ - and S-doped graphene or metal clusters on graphene. It would be interesting to characterize water adsorption on these systems using surface science techniques.

\section{CONCLUSIONS}

A short literature survey is provided about water interacting with graphene, focusing on experimental surface science works conducted at UHV. Discussed is how a readily available 
experimental surface science technique can be used to determine wetting properties of water on graphene at clean vacuum conditions. Data on ultimately clean samples are, however, limited to graphene that can actually be made at UHV.

\section{Acknowledgments}

The published results of my group which are also described in this review were related to the $\mathrm{PhD}$ research of Ashish Chakradhar, Mudivans Tilan Nayakasinghe, and Nilushni Sivapragasam at NDSU. Their support, discussions, and work effort in the prior projects are acknowledged. The donors of the American Chemical Society and Petroleum Research Fund are acknowledged for partial financial support of our prior work on water/graphene. The students also were supported by teaching assistantships at NDSU.

\section{References}

1. C.-H. Chang, M.-H. Hsu, C.-J. Weng, C.-W. Peng, W.-I. Hung, K.-C. Chang, T.-L. Chuang, Y.-C. Yen, and J.-M. Yeh: 3D-bioprinting approach to fabricate superhydrophobic epoxy/ organophilic clay as advanced anticorrosive coatings with a synergistic effect of superhydrophobicity and gas barrier property. J. Mater. Chem. A 1, 13869-13877 (2013).

2. M.J. Kreder, J. Alvarenga, P. Kim, and J. Aizenberg: Design of anti-icing surfaces: Smooth, textured or slippery? Nat. Rev. Mater. 1, 1 (2016).

3. A. Chakradhar and U. Burghaus: Adsorption of water on graphene/Ru(0001) - An experimental ultra-high vacuum study. Chem. Commun. 50, 7698-7701 (2014).

4. A. Chakradhar, N. Sivapragasam, M.T. Nayakasinghe, and U. Burghaus: Support effects in the adsorption of water on CVD graphene: An ultra-high vacuum adsorption study. Chem. Commun. 51, 11463-11466 (2015).

5. J. Shan, A. Chakradhar, Z. Yu, and U. Burghaus: Adsorption of water on a hydrophobic surface - The case of antimony(111). Chem. Phys. Lett. 517, 46-50 (2011).

6. U. Burghaus: Adsorption of water on two-dimensional crystals: Water/graphene and water/silicatene (short review). Inorganics 4 , 10 (2016).

7. M. Komarneni, A. Sand, J. Goering, U. Burghaus, M. Lu, M. Veca, and Y.-P. Sun: Possible effect of carbon nanotube diameter on gas-surface interactions - The case of benzene, water, and n-pentane adsorption on SWCNTs at ultra-high vacuum conditions. Chem. Phys. Lett. 476, 227-231 (2009).

8. N. Sivapragasam, M.T. Nayakasinghe, and U. Burghaus: Adsorption kinetics and dynamics of $\mathrm{CO}_{2}$ on $\mathrm{Ru}(0001)$ supported graphene oxide. J. Phys. Chem. C 120, 28049-28056 (2016).
9. A. Chakradhar, N. Sivapragasam, M.T. Nayakasinghe, and U. Burghaus: Adsorption kinetics of benzene on graphene: An ultra-high vacuum study. J. Vac. Sci. Technol. A 34, 021402 (2016).

10. N. Sivapragasam, M.T. Nayakasinghe, and U. Burghaus: Adsorption of $\mathrm{n}$-butane on graphene/Ru(0001) - A molecular beam scattering study. J. Vac. Sci. Technol. 34, 041404 (2016).

11. A. Chakradhar, K.M. Trettel, and U. Burghaus: Benzene adsorption on $\mathrm{Ru}(0001)$ and graphene $/ \mathrm{Ru}(0001)$ - How to synthesize epitaxial graphene without STM or LEED? Chem. Phys. Lett. 590, 146-152 (2013).

12. N. Sivapragasam, M.T. Nayakasinghe, A. Chakradhar, and U. Burghaus: Effects of the support on the desorption kinetics of n-pentane from graphene: An ultra-high vacuum adsorption study. J. Vac. Sci. Technol. A 35, 061404 (2017).

13. U. Burghaus: Gas-surface interactions on two-dimensional crystals. Surf. Sci. Rep. 74, 141-177 (2019).

14. U. Burghaus: Gas-carbon nanotubes interactions: a review of ultra-high vacuum surface science studies on CNTs. In Carbon Nanotubes - Research Trends (Nova Science, New York). ISBN 978-1-60692-236-1 (2009).

15. P.A. Thiel and T.E. Madey: The interaction of water with solid surfaces: Fundamental aspects. Surf. Sci. Rep. 7, 211 (1987).

16. M.A. Henderson: The interaction of water with solid surfaces: Fundamental aspects revisited. Surf. Sci. Rep. 46, 1 (2002).

17. R.S. Smith and B.D. Kay: Molecular beam studies of kinetic processes in nanoscale water films. Surf. Rev. Lett. 4, 781 (1997).

18. C.J. Shih, M.S. Strano, and D. Blankschtein: Wetting translucency of graphene. Nat. Mater. 12, 866 (2013).

19. J. Rafiee, X. Mi, H. Gullapalli, A.V. Thomas, F. Yavari, Y. Shi, P.M. Ajayan, and N.A. Koratkar: Wetting transparency of graphene. Nat. Mater. 11, 217 (2012).

20. C.T. Campbell: Ultrathin metal films and particles on oxide surfaces: Structural, electronic and chemisorptive properties. Surf. Sci. Rep. 27, 1 (1997).

21. V.A. Bakaev and W.A. Steele: On the computer simulation of a hydrophobic vitreous silica surface. J. Chem. Phys. 111, 9803 (1999).

22. G.A. Morgan, D.C. Sorescu, T. Zubkov, and J.T. Yates, Jr.: The formation and stability of adsorbed formyl as a possible intermediate in Fischer-Tropsch chemistry on ruthenium. J. Phys. Chem. B 108, 3614-3624 (2004).

23. H. Niemelä-Anttonen, H. Koivuluoto, M. Tuominen, H. Teisala, P. Juuti, J. Haapanen, J. Harra, C. Stenroos, J. Lahti, J. Kuusipalo, J.M. Mäkelä, and P. Vuoristo: Icephobicity of slippery liquid infused porous surfaces under multiple freeze-thaw and ice accretion-detachment cycles. Adv. Mater. Interfaces 5, 1800828 (2018).

24. P. Irajizad, S. Nazifi, and H. Ghasemi: Historical perspective, icephobic surfaces: Definition and figures of merit. Adv. Colloid Interface Sci. 269, 203-218 (2019). 
25. S. Jung, M. Dorrestijn, D. Raps, A. Das, C.M. Megaridis, and D. Poulikakos: Are superhydrophobic surfaces best for icephobicity? Langmuir 27, 3059-3066 (2011).

26. A.J. Meuler, D. Smith, K.K. Varanasi, J.M. Mabry, G.H. McKinley, and R.E. Cohenet al.: Relationships between water wettability and ice adhesion. ACS Appl. Mater. Interfaces 11, $3100-3110(2010)$

27. V. Hejazi, K. Sobolev, and M. Nosonovsky: From superhydrophobicity to icephobicity: Forces and interaction analysis. Sci. Rep. 3, 2194 (2013).

28. N. Severin, M. Dorn, A. Kalachev, and J.P. Rabe: Replication of single macromolecules with graphene. Nano Lett. 11, 2436-2439 (2011).

29. E. Lundgren, G. Kresse, C. Klein, M. Borg, J.N. Andersen, M.D. Santis, Y. Gauthier, C. Konvicka, M. Schmid, and P. Varga: Two-dimensional oxide on Pd(111). Phys. Rev. Lett. 88, 246103 (2002).

30. O. Leenaerts, B. Partoens, and F.M. Peeters: Adsorption of $\mathrm{H}_{2} \mathrm{O}$, $\mathrm{NH}_{3}, \mathrm{CO}, \mathrm{NO}_{2}$, and $\mathrm{NO}$ on graphene: A first-principles study. Phys. Rev. B 77, 125416 (2008).

31. J. Ma, A. Michaelides, D. Alf, L. Schimka, G. Kresse, and E. Wang: Adsorption and diffusion of water on graphene from first principles. Phys. Rev. B 84, 033402 (2011).

32. J. Kysilka, M. Rube, L. Grajciar, P. Nachtigall, and O. Bludsk: Accurate description of argon and water adsorption on surfaces of graphene-based carbon allotropes. J. Phys. Chem. A 115, 11387 (2011).

33. J. Wang and L. Wang: Superhydrophilic and underwater superoleophobic nanofibrous membrane for separation of oil/water emulsions. J. Mater. Res. 35, 1504-1513 (2020).

34. J.C. Liu, P.A. Monson, and F.v. Swol: Studies of a lattice model of water confined in a slit pore. J. Phys. Chem. C 111, 15976-15981 (2007).

35. C. Gründling, J.A. Lercher, and D.W. Goodman: Preparation of mixed $\mathrm{Al}_{2} \mathrm{O}_{3} / \mathrm{SiO}_{2}$ thin films supported on $\mathrm{Mo}(100)$. Surf. Sci. 318 97-103 (1994).

36. J.S. Qiang Li, F. Besenbacher, and M. Dong: Two-dimensional material confined water. Acc. Chem. Res. 48, 119-127 (2015).

37. P. Bampoulis, K. Sotthewes, E. Dollekamp, and B. Poelsema: Water confined in two-dimensions: Fundamentals and applications. Surf. Sci. Rep. 73, 233-264 (2018).

38. K. Xu, P. Cao, and J.R. Heath: Graphene visualizes the first water adlayers on mica at ambient conditions. Science 329, 1188-1191 (2010).

39. C.J. Shih, Q.H. Wang, S. Lin, K.C. Park, Z. Jin, M.S. Strano, and D. Blankschtein: Breakdown in the wetting transparency of graphene. Phys. Rev. Lett. 109, 176101 (2012).

40. M.J. Gillan, D. Alfè, and A. Michaelides: Perspective: How good is DFT for water? J. Chem. Phys. 144, 130901 (2016).

41. Z. Li, A.W. Lepore, M.F. Salazar, G.S. Foo, B.H. Davison,

Z. Wu, and C.K. Narula: Selective conversion of bio-derived ethanol to renewable BTX over Ga-ZSM-5. Green Chemistry 19, 4344-4352 (2017).

42. F. Jiao and H. Frei: Nanostructured cobalt oxide clusters in mesoporous silica as efficient oxygen-evolving catalysts. Angew. Chem. Int. Ed. Engl. 48, 1841-1844 (2009).

43. M. Moshoeshoe, M.S. Nadiye-Tabbiruka, and V. Obuseng: Properties and applications of zeolites. Am. J. Mater. Sci. 7, 196221 (2017).

44. R. Raj, S.C. Maroo, and E.N. Wang: Wettability of graphene. Nano Lett. 13, 1509-1515 (2013).

45. N. Kosinov, J. Gascon, F. Kapteijn, and E.J. Hensen: Recent developments in zeolite membranes for gas separation. J. Memb. Sci. 499, 65-79 (2016).

46. T. Shirai, H. Watanabe, M. Fuji, and M. Takahashi: Structural properties and surface characteristics on aluminum oxide powders. Nagoya Inst. Technol. 9, 23-31 (2009).

47. T.O. Wehling, A.I. Lichtenstein, and M.I. Katsnelson: First-principles studies of water adsorption on graphene: The role of the substrate. Appl. Phys. Lett. 93 (2008).

48. V.M. Bermudez and J.T. Robinson: Effects of molecular adsorption on the electronic structure of single-layer graphene. Langmuir 27, 11026-11036 (2011).

49. Z. Wang and C.J. Liu: Preparation and application of iron oxide/ graphene based composites for electrochemical energy storage and energy conversion devices: Current status and perspective. Nano Energy 11, 277 (2015).

50. X. Feng, S. Maier, and M. Salmeron: Water splits epitaxial graphene and intercalates. J. Am. Chem. Soc. 134, 5662-5668 (2012).

51. Z. Li, Y. Wang, A. Kozbial, G. Shenoy, F. Zhou, R. McGinley,

P. Ireland, B. Morganstein, A. Kunkel, S.P. Surwade, L. Li, and H. Liu: Study on the surface energy of graphene by contact angle measurements. Nat. Mater. 12, 925 (2013).

52. P. Cao, K. Xu, J.O. Varghese, and J.R. Heath: The microscopic structure of adsorbed water on hydrophobic surfaces under ambient conditions. Nano Lett. 11, 5581-5586 (2011).

53. Z. Li, Y. Wang, A. Kozbial, G. Shenoy, F. Zhou, R. McGinley, P. Ireland, B. Morganstein, A. Kunkel, S.P. Surwade, L. Li, and H. Liu: Effect of airborne contaminants on the wettability of supported graphene and graphite. Nat. Mater. 12, 925-931 (2013).

54. S. Sahoo, S.L. Suib, and S.P. Alpay: Graphene supported single atom transition metal catalysts for methane activation. ChemCatChem 10, 3229-3235 (2018).

55. S. Singla, E. Anim-Danso, A.E. Islam, Y. Ngo, S.S. Kim, R.R. Naik, and A. Dhinojwala: Insight on structure of water and ice next to graphene using surface-sensitive spectroscopy. ACS Nano 11, 4899-4906 (2017).

56. E. Voloshina, D. Usvyat, M. Schutz, Y. Dedkovcd, and B. Paulusa: On the physisorption of water on graphene: A CCSD (T) study. Phys. Chem. Chem. Phys. 13, 12041-12047 (2011). 
57. D.V. Chakarov, L. Osterlund, and B. Kasemo: Water adsorption and coadsorption with potassium on graphite(0001). Langmuir 11, 1201-1214 (1995).

58. D.V. Chakarov, L. Osterlund, and B. Kasemo: Water adsorption on graphite (0001). Vacuum 46, 1109-1112 (1995).

59. B.D. Kay, K.R. Lykke, J.R. Creighton, and S.J. Ward: The influence of adsorbate-adsorbate hydrogen bonding in molecular chemisorption: $\mathrm{NH}_{3}, \mathrm{HF}$, and $\mathrm{H}_{2} \mathrm{O}$ on $\mathrm{Au}(111)$. J. Chem. Phys. 91, 5120-5221 (1989).

60. A. Hodgson and S. Haq: Water adsorption and the wetting of metal surfaces. Surf. Sci. Rep. 64, 381-451 (2009).

61. T.T. Song, M. Yang, J.W. Chai, M. Callsen, J. Zhou, T. Yang, Z. Zhang, J.S. Pan, D.Z. Chi, Y.P. Feng, and S.J. Wang: The stability of aluminium oxide monolayer and its interface with two-dimensional materials. Sci. Rep. 6, 29221 (2016).

62. S. Hu, M. Lozada-Hidalgo, F.C. Wang, A. Mishchenko, F. Schedin, R.R. Nair, E.W. Hill, D.W. Boukhvalov, M.I. Katsnelson, R.A.W. Dryfe, I.V. Grigorieva, H.A. Wu, and A.K. Geim: Proton transport through one-atom-thick crystals. Nature 516, 227-230 (2014).

63. M. Zhou, A. Zhang, Z. Dai, C. Zhang, and Y.P. Feng: Greatly enhanced adsorption and catalytic activity of $\mathrm{Au}$ and Pt clusters on defective graphene. J. Chem. Phys. 132, 194704 (2010).

64. A. Ambrosetti and P.L. Silvestrelli: Communication: Enhanced chemical reactivity of graphene on a $\mathrm{Ni}(111)$ substrate. J. Chem. Phys. 144, 111101 (2016).

65. T.R. Linderoth, V.P. Zhdanov, and B. Kasemo: Water condensation kinetics on a hydrophobic surface. Phys. Rev. Lett. 90, 156103 (2003).

66. B.J. Hinch and L.H. Dubois: Stable and metastable phases of water adsorbed on Cu(111). J. Chem. Phys. 96, 3262 (1992).

67. S. Srivastava, P.K. Kashyap, V. Singh, T.D. Senguttuvan, and B.K. Gupta: Nitrogen doped high quality CVD grown graphene as a fast responding $\mathrm{NO}_{2}$ gas sensor. New J. Chem. 42, 9550-9556 (2018).

68. Y.W. Chen and H.P. Cheng: Interaction between water and defective silica surfaces. J. Chem. Phys. 134, 114703 (2011).

69. R.G. Quiller, T.A. Baker, X. Deng, M.E. Colling, B.K. Min, and C.M. Friend: Transient hydroxyl formation from water on oxygen-covered Au(111). J. Chem. Phys. 129, 064702 (2008).

70. M.T. Nayakasinghe, Y. Han, N. Sivapragasam, D. Kilin, N. Oncel, and U. Burghaus: Adsorption of formic acid on $\mathrm{CH}_{3} \mathrm{NH}_{3} \mathrm{PbI}_{3}$ lead-halide organic-inorganic perovskites. J. Phys. Chem. C 123, 22873-22886.

71. A. Almenningen, O. Bastiansen, V. Ewing, K. Hedberg, and

M. Traetteberg: The molecular structure of disiloxane, $\left(\mathrm{SiH}_{3}\right)_{2} \mathrm{O}$. Acta Chem. Scand. 17, 9 (1963).
72. R. Liu, T. Gong, K. Zhang, and C. Lee: Graphene oxide papers with high water adsorption capacity for air dehumidification. Sci. Rep. 7, 9761 (2017).

73. B. Lian, S.D. Luca, Y. You, S. Alwarappan, M. Yoshimura, V. Sahajwalla, S.C. Smith, G. Leslie, and R.K. Joshi: extraordinary water adsorption characteristics of graphene oxide. Chem. Sci. 9, 5106 (2018).

74. K. Zhou, K.S. Vasu, and C.T. Cherian: Electrically controlled water permeation through graphene oxide membranes. Nature 559, 236-240 (2018).

75. F. Mouhat, F. Coudert, and M. Bocquet: Structure and chemistry of graphene oxide in liquid water from first principles. Nat. Commun. 11, 1566 (2020).

76. C. Lin, W. Wei, and Y.H. Hu: Catalytic behavior of graphene oxide for cement hydration process. J. Phys. Chem. Solids 89 , 128-133 (2016).

77. J. Shan, J.F.M. Aarts, A.W. Kleyn, and L.B.F. Juurlink: Co-adsorption of water and hydrogen on Ni(111). Phys. Chem. Chem. Phys. 10, 4994-5003 (2008).

78. M.J.T.C. van der Niet, I. Dominicus, M.T.M. Koper, and L.B.F. Juurlink: Hydrophobic interactions between water and pre-adsorbed D on the stepped $\operatorname{Pt}(533)$ surface. Phys. Chem. Chem. Phys. 10, 7169-7179 (2008).

79. G.A. Kimmel, N.G. Petrik, Z. Dohnalek, and B.D. Kay: Crystalline ice growth on $\mathrm{Pt}(111)$ : Observation of a hydrophobic water monolayer. Phys. Rev. Lett. 95, 166102 (2005).

80. K.J. Wu, L.D. Peterson, G.S. Elliott, and S.D. Kevan: Time-resolved electron energy loss spectroscopy study of water desorption from $\operatorname{Ag}(011)$. J. Chem. Phys. 91, 7964 (1989).

81. A.S. Bolina, A.J. Wolff, and W.A. Brown: Reflection absorption infrared spectroscopy and temperature-programmed desorption studies of the adsorption and desorption of amorphous and crystalline water on a graphite surface. J. Phys. Chem. B 109, 16836-16845 (2005).

82. R. Souda: Nanoconfinement effects of water on hydrophilic and hydrophobic substrates at cryogenic temperatures. J. Phys. Chem. C 116, 20895-20901 (2012).

83. M.T. Nayakasinghe, N. Sivapragasam, and U. Burghaus: Desulfurization-related surface chemistry on two-dimensional silica films: Adsorption of thiophene and short-chain alkanes on silicatene. J. Phys. Chem. C 122, 8244-8253 (2018).

84. G.A. Kimmel, J. Matthiesen, M. Baer, C.J. Mundy, N.G. Petrik, R.S. Smith, Z. Dohnalek, and B.D. Kay: No confinement needed: observation of a metastable hydrophobic wetting two-layer ice on graphene. J. Am. Chem. Soc. 131, 12838-12844 (2009).

85. T. Lim, and S. Ju: Control of graphene surface wettability by using CF4 plasma. Surf. Coating. Technol. 328, 89-93 (2017). 